ISSN 1112-9867

Special Issue

http://www.jfas.info

\title{
NEWTON-SOR ITERATIVE METHOD FOR SOLVING THE TWO-DIMENSIONAL POROUS MEDIUM EQUATION
}

\author{
J. V. L. Chew ${ }^{*}$ and J. Sulaiman \\ Faculty of Science and Natural Resources, Universiti Malaysia Sabah, 88400 Kota Kinabalu, \\ Sabah, Malaysia
}

Published online: 10 November 2017

\begin{abstract}
In this paper, we consider the application of the Newton-SOR iterative method in obtaining the approximate solution of the two-dimensional porous medium equation (2D PME). The nonlinear finite difference approximation equation to the 2D PME is derived by using the implicit finite difference scheme. The developed nonlinear system is linearized by using the Newton method. At each temporal step, the corresponding linear systems are solved by using SOR iteration. We investigate the efficiency of the Newton-SOR iterative method by solving three examples of 2D PME and the performance is compared with the Newton-GS iterative method. Numerical results show that the Newton-SOR iterative method is better than the Newton-GS iterative method in terms of a number of iterations, computer time and maximum absolute errors.
\end{abstract}

Keywords: porous medium equation; finite difference scheme; Newton; Successive Over Relaxation, Gauss-Seidel.

Author Correspondence, e-mail: jackelchew93@gmail.com

doi: http://dx.doi.org/10.4314/jfas.v9i6s.30 


\section{INTRODUCTION}

Mathematical models that are developed in order to describe many natural and engineering phenomena are generally written in the forms of partial differential equation (PDE). The phenomena are usually modeled and described together with the appropriate initial and boundary conditions. The solution of the mathematical models can be obtained with respect to the given initial and boundary conditions.

With exceptional to the case where the exact solution can be obtained, most of the nonlinear PDE problems are difficult to be solved. The solution of the nonlinear heat and mass transfer problem, for instance plays a significant role in the study of various thermal and diffusion processes. It makes the study of the mechanisms in the particular complex physical phenomena easier to be conducted. In addition to that, a particular exact solution of PDE problem can be used to verify the accuracy and efficiency of various numerical methods. In the other words, PDE problems with exact solutions can be used in the experiment of developing new numerical methods which in turn enable researchers to study more complex PDE problems [1].

This paper considers the application of the Newton-Successive Over Relaxation (Newton-SOR) iterative method for solving the two-dimensional porous medium equation (2D PME) under the bounded spatial region $\Omega=[0,1] \times[0,1]$ and time interval $t=(0,1)$ which can be defined as [2]:

$$
\begin{aligned}
& \frac{\partial u}{\partial t}=\alpha\left[\frac{\partial}{\partial x}\left(u^{m} \frac{\partial u}{\partial x}\right)+\frac{\partial}{\partial y}\left(u^{m} \frac{\partial u}{\partial y}\right)\right], u(x, y, t)=g(x, y, t),(x, y) \in \Omega \\
& u(x, y, 0)=u_{0}(x, y) .
\end{aligned}
$$

where $m$ and $\alpha$ are rational numbers, satisfying the exact solution $g(x, y, t)$ and subject to the initial condition $u_{0}(x, y)$.

From the recent literature survey on the application and the solution of Equation (1), in [3] studied the role of transient solidification processing parameters for the designing of the microstructure in the continuous casting process. They employed the inverse heat conduction method to solve the heat flow across the metal casting/mould interface phenomena, which is characterized by a macroscopic average metal/mould interfacial heat transfer coefficient based 
on the measure of the temperature inside the casting. They used the explicit finite difference scheme onto the set of boundary conditions and the heat conduction equation that resembles Equation (1). The heat flow problem is solved by minimizing the standard least square norm between the estimated temperatures computed from the solution of the direct problem in which using the estimated values of the heat transfer coefficient components and the experimental temperatures.

In [4] studied the solution of the instability phenomenon based on the double phase flow of two immiscible fluids through the inclined homogeneous porous medium. They implemented the Crank-Nicolson finite difference scheme onto the derived nonlinear PDE that similar with the one-dimensional case of Equation (1) and solved it numerically.

Other than the use of finite difference scheme,Equation (1) which can also be known as the heat and mass transfer equation with power-law temperature-dependent thermal conductivity was studied by [5]. They applied an analytical approach called the Adomian's decomposition method for the solution. Besides that, the one-dimensional case of the PME problem has been studied numerically by [6-7] with the use finite difference scheme and iterative methods.

This paper aims to solve Equation (1) with the use finite difference scheme together with the application of the Newton-SOR iterative method. This study investigates the efficiency of the applied numerical technique in terms of the number of iterations, the computer time and the maximum absolute errors over the Newton-Gauss-Seidel (Newton-GS) iterative method [8].

\section{FINITE DIFFERENCE APPROXIMATION EQUATION}

In this paper, we discretize $u(x, y, t)$ on an equidistant spatial-temporal grid $\left(x_{i}, y_{j}, t_{n}\right)=(i h, j h, n k)$ with $1 \leq i, j \leq M, 0<n<T$ where $h=\Delta x=\Delta y$ and $k=\Delta t$ are spatial and temporal steps respectively. The implicit finite difference scheme is used due to its good stability and yields lesser computational cost compared to Crank-Nicolson scheme. When Equation (1) is discretized by using the implicit finite difference scheme, the resultant finite difference approximation equation can be written as 


$$
\begin{aligned}
& f\left(u_{i, j, n+1}\right)=u_{i, j, n+1}-A_{1}\left(u_{i, j, n+1}^{m} u_{i+1, j, n+1}-2 u_{i, j, n+1}^{m+1}+u_{i, j, n+1}^{m} u_{i-1, j, n+1}\right) \\
& -A_{2}\left(m u_{i, j, n+1}^{m-1} u_{i+1, j, n+1}^{2}-2 m u_{i, j, n+1}^{m-1} u_{i+1, j, n+1} u_{i-1, j, n+1}+m u_{i, j, n+1}^{m-1} u_{i-1, j, n+1}^{2}\right) \\
& -A_{3}\left(u_{i, j, n+1}^{m} u_{i, j+1, n+1}-2 u_{i, j, n+1}^{m+1}+u_{i, j, n+1}^{m} u_{i, j-1, n+1}\right) \\
& -A_{4}\left(m u_{i, j, n+1}^{m-1} u_{i, j+1, n+1}^{2}-2 m u_{i, j, n+1}^{m-1} u_{i, j+1, n+1} u_{i, j-1, n+1}+m u_{i, j, n+1}^{m-1} u_{i, j-1, n+1}^{2}\right)-u_{i, j, n}
\end{aligned}
$$

where $A_{1}=A_{3}=\frac{\alpha k}{(h)^{2}}, A_{2}=A_{4}=\frac{\alpha k}{4(h)^{2}}$ with $k=\frac{1}{T}$ and $h=\frac{1}{M}$.

The implementation of Equation (2) to every interior mesh point in region $\Omega$ will result a sparse and large system of nonlinear equations as it relates every neighborhood points. Such nonlinear system can be generally written as

$$
F(\underset{\sim}{U})=0
$$

$$
\begin{aligned}
& \text { where } \underset{\sim}{F}(\underset{\sim n+1}{U})=\left(f_{1,1}, f_{2,1}, \ldots, f_{1,2}, f_{2,2}, \ldots, f_{M, M}\right)^{T} \\
& \underset{\sim n+1}{U}=\left(u_{1,1, n+1}, u_{2,1, n+1}, \ldots, u_{1,2, n+1}, u_{2,2, n+1}, \ldots, u_{M, M, n+1}\right) .
\end{aligned}
$$

and

Solving Equation(3) generally will face the high complexity problem. Therefore, it needs an efficient iterative method to be solved.

\section{NEWTON-SOR ITERATIVE METHOD}

In the year 1967, in [8] have studied the monotone iteration processes for the nonlinear elliptic boundary value problems and introduced the Newton-GS iterative method. This method uses the Gauss-Seidel iteration on the linear systems of Newton's method or the linearized system of equations. They have suggested that by using the optimum relaxation parameter of $\omega>1$ on Newton-GS iterative method, a faster convergence of the linearized system of equations can be achieved. More in [9] has extended the study of the convergence properties of the Newton-GS iterative method and produced the global convergence theorems for the Newton-GS iterative method and its related methods.

Actually, when a relaxation parameter $1<\omega<2$ is applied onto the Newton-GS iterative method such method can be extended and called as the Newton-SOR iterative method. The concept of the SOR iteration was introduced by [10].

Now, Newton-SOR iterative method will be applied to solve Equation (3). To begin, Newton method is used to transform Equation (3) into the corresponding linear system as follows: 


$$
J_{F}\left(\underset{\sim n+1}{U_{(\ell)}^{(\ell)}}\right) \underset{\sim}{\Delta H_{n+1}^{(\ell)}}=-\underset{\sim}{\sim}\left(\underset{\sim n+1}{U_{(\ell)}^{(\ell)}}\right), \ell=1,2, \ldots
$$

where $J_{F}\left(\underset{\sim n+1}{U_{(\ell)}^{(\ell)}}\right)$ is a pentadiagonal $M^{2} \times M^{2}$ Jacobian matrix, $\underset{\sim}{\Delta H_{n+1}^{(\ell)}}$ and $-\underset{\sim}{F}\left(\underset{\sim n+1}{U_{(\ell)}^{(\ell)}}\right)$ are column matrices with iterative index $(\ell)$ and time level $n+1$.

Let the matrix $J_{F}\left(\underset{\sim n+1}{U^{(\ell)}}\right)$ be decomposed into

$$
J_{F}\left(\underset{\sim n+1}{U_{(\ell)}^{(\ell)}}\right)=D-L-V
$$

By using Equation (5), the SOR iteration can be implemented onto Equation (4) and the resultant Newton-SOR iterative method can be written as

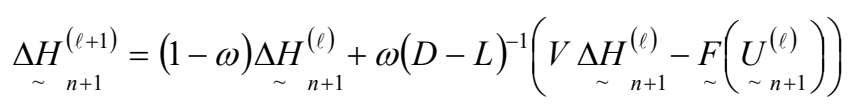

where $\omega$ represents the relaxation parameter and $\underset{\sim}{\Delta H_{n+1}^{(\ell+1)}}=\underset{\sim n+1}{U_{(\ell+1)}^{(\ell)}}-\underset{\sim n+1}{U^{(\ell)}}$.

In practice, the optimum value of $\omega$ is determined by running the Algorithm 1 several times until the least number of iterations is obtained. Moreover, when $\omega=1$, the Newton-SOR iterative method reverts back to the Newton-GS iterative method.

\section{Algorithm 1: Newton-SOR Iterative Method}

i. Compute $A_{1}, A_{2}, A_{3}$, and $A_{4}$,

ii. $\quad$ Compute $-\underset{\sim}{F}\left(\underset{\sim n+1}{U_{\sim}^{(\ell)}}\right)$ and $J_{F}\left(\underset{\sim n+1}{U_{\sim}^{(\ell)}}\right)$,

iii. Iterate Eq. (6),

iv. $\quad$ If $\left|\underset{\sim}{\Delta H_{n+1}^{(\ell+1)}}-\underset{\sim}{\Delta H_{n+1}^{(\ell)}}\right|<\varepsilon$, compute the solution vectors $\underset{\sim n+1}{U_{(\ell+1)}^{(\ell)}}=\underset{\sim}{\Delta H_{n+1}^{(\ell+1)}}+\underset{\sim n+1}{U^{(\ell)},}$

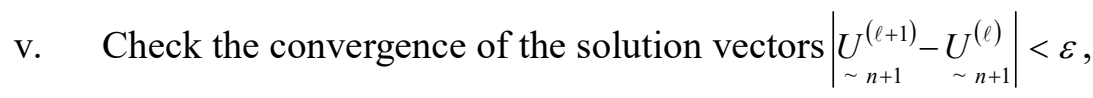

vi. Go to the next temporal step.

\section{NUMERICAL EXPERIMENTS}

In this section, numerical results are presented in order to observe the performance and the efficiency of the proposed Newton-SOR iterative method against the control Newton-GS iterative method. Three parameters which are the number of iterations $(\ell)$, the computer time in seconds $(s)$ and the maximum absolute errors (error) will be measured for a comparative 
analysis between the Newton-SOR and the Newton-GSiterative methods. The following three 2D PME are used as the test problems.

\section{Example 1 [2]:}

We choose $\alpha=0.2$ and $m=1$ in Equation (1) becomes

$$
\frac{\partial u}{\partial t}=0.2\left[\frac{\partial}{\partial x}\left(u \frac{\partial u}{\partial x}\right)+\frac{\partial}{\partial y}\left(u \frac{\partial u}{\partial y}\right)\right] .
$$

and the exact solution is given by $u(x, y, t)=x+y+(0.4) t$.

\section{Example 2 [2]:}

We take $\alpha=0.2$ and $m=2$, and Equation (1) becomes

$$
\frac{\partial u}{\partial t}=0.2\left[\frac{\partial}{\partial x}\left(u^{2} \frac{\partial u}{\partial x}\right)+\frac{\partial}{\partial y}\left(u^{2} \frac{\partial u}{\partial y}\right)\right] .
$$

and the exact solution is given by $u(x, y, t)=(5(x+y+t))^{1 / 2}$.

\section{Example 3 [11]:}

We use $\alpha=1.0$ and $m=5$ and the problem becomes

$$
\frac{\partial u}{\partial t}=\frac{\partial}{\partial x}\left(u^{5} \frac{\partial u}{\partial x}\right)+\frac{\partial}{\partial y}\left(u^{5} \frac{\partial u}{\partial y}\right)
$$

and the exact solution is $u(x, y, t)=\left(\frac{4(x+y+2 t)}{5}\right)^{1 / 4}$.

Throughout the simulations, the convergence parameter is set to be $\varepsilon=10^{-10}$ and the size of matrices to be considered are 16, 32, 64, 128 and 256. The numerical results for the three examples are tabulated in Tables 1, 2 and 3. 
Table 1.Comparison of number of iterations $(\ell)$, computer time in seconds $(s)$ and maximum absolute error (error) for Example 1

\begin{tabular}{cccccc}
\hline M & Method & $\omega$ & $(\ell)$ & $(s)$ & Error \\
\hline \multirow{2}{*}{16} & GS & 1.00 & 136 & 0.35 & $8.87 \mathrm{E}-11$ \\
& SOR & 1.19 & 100 & 0.28 & $3.93 \mathrm{E}-12$ \\
32 & GS & 1.00 & 438 & 2.35 & $2.97 \mathrm{E}-10$ \\
& SOR & 1.43 & 207 & 1.43 & $1.59 \mathrm{E}-11$ \\
64 & GS & 1.00 & 1526 & 19.77 & $1.88 \mathrm{E}-09$ \\
& SOR & 1.64 & 412 & 8.47 & $5.83 \mathrm{E}-11$ \\
128 & GS & 1.00 & 5459 & 258.55 & $8.96 \mathrm{E}-09$ \\
& SOR & 1.80 & 813 & 55.72 & $8.16 \mathrm{E}-11$ \\
256 & GS & 1.00 & 19388 & 4275.66 & $3.85 \mathrm{E}-08$ \\
& SOR & 1.89 & 1608 & 454.53 & $1.44 \mathrm{E}-10$ \\
\hline
\end{tabular}

Table 2. Comparison of number of iterations $(\ell)$, computer time in seconds $(s)$ and maximum absolute error (error) for Example 2

\begin{tabular}{|c|c|c|c|c|c|}
\hline $\mathbf{M}$ & Method & $\omega$ & $(\ell)$ & $(s)$ & Error \\
\hline & GS & 1.00 & 130 & 0.47 & $7.57 \mathrm{E}-11$ \\
\hline & SOR & 1.16 & 100 & 0.30 & $2.58 \mathrm{E}-11$ \\
\hline & GS & 1.00 & 400 & 1.96 & $2.31 \mathrm{E}-09$ \\
\hline & SOR & 1.37 & 208 & 1.48 & $1.26 \mathrm{E}-10$ \\
\hline & GS & 1.00 & 1380 & 19.26 & $1.31 \mathrm{E}-08$ \\
\hline & SOR & 1.60 & 420 & 8.68 & $2.97 \mathrm{E}-10$ \\
\hline & GS & 1.00 & 4901 & 248.82 & $4.95 \mathrm{E}-08$ \\
\hline & SOR & 1.77 & 836 & 56.86 & $5.66 \mathrm{E}-10$ \\
\hline & GS & 1.00 & 17458 & 4243.79 & $1.75 \mathrm{E}-07$ \\
\hline & SOR & 1.87 & 1658 & 478.09 & $1.50 \mathrm{E}-09$ \\
\hline
\end{tabular}


Table 3. Comparison of number of iterations $(\ell)$, computer time in seconds $(s)$ and maximum absolute error (error) for Example 3

\begin{tabular}{cccccc}
\hline M & Method & $\omega$ & $(\ell)$ & $(s)$ & Error \\
\hline 16 & GS & 1.00 & 739 & 0.82 & $1.10 \mathrm{E}-09$ \\
& SOR & 1.55 & 291 & 0.50 & $2.84 \mathrm{E}-11$ \\
32 & GS & 1.00 & 2630 & 8.51 & $6.69 \mathrm{E}-09$ \\
& SOR & 1.74 & 573 & 2.92 & $3.97 \mathrm{E}-11$ \\
64 & GS & 1.00 & 9478 & 113.63 & $3.56 \mathrm{E}-08$ \\
& SOR & 1.86 & 1118 & 18.86 & $5.69 \mathrm{E}-11$ \\
128 & GS & 1.00 & 34098 & 1653.85 & $1.72 \mathrm{E}-07$ \\
& SOR & 1.93 & 2178 & 131.63 & $6.65 \mathrm{E}-11$ \\
& GS & 1.00 & 121649 & 29234.80 & $7.78 \mathrm{E}-07$ \\
& SOR & 1.96 & 4276 & 1163.89 & $6.26 \mathrm{E}-11$ \\
\hline
\end{tabular}

Based on Table 1, it can be observed that with the increasing size of matrices $M$, the Newton-SOR iterative method exhibits a lesser number of iterations and faster computer time compared to the Newton-GS iterative method when it is at the optimal relaxation parameter. Then, we have computed the percentage of improvement in terms of a number of iterations $(\Delta \ell) \%$ and computer time $(\Delta s) \%$ by the Newton-SOR iterative method against the Newton-GS iterative method using the expressions:

$$
(\Delta \ell) \%=\left|\frac{\ell_{G S}-\ell_{S O R}}{\ell_{G S}}\right| \times 100 \%
$$

and

$$
(\Delta s) \%=\left|\frac{s_{G S}-s_{S O R}}{s_{G S}}\right| \times 100 \%,
$$

and the result is tabulated in Table 4. 
Table 4.Percentage of improvement in terms of number of iterations $(\Delta \ell) \%$ and computer time $(\Delta s) \%$ by the Newton-SOR method against the Newton-GS method

\begin{tabular}{cc}
\hline M & Number of iterations $(\Delta \ell) \%$ \\
\hline 16 & $23.08 \%-60.62 \%$ \\
32 & $48.00 \%-78.21 \%$ \\
64 & $69.57 \%-88.20 \%$ \\
128 & $82.94 \%-93.61 \%$ \\
256 & $90.50 \%-96.48 \%$ \\
\hline M & Computer time $(\Delta s) \%$ \\
\hline 16 & $20.00 \%-39.02 \%$ \\
32 & $24.49 \%-65.69 \%$ \\
64 & $54.93 \%-83.40 \%$ \\
128 & $77.15 \%-92.04 \%$ \\
256 & $88.73 \%-96.02 \%$ \\
\hline
\end{tabular}

\section{CONCLUSION}

This paper investigates the application and the efficiency of the Newton-SOR iterative method in obtaining the approximate solution of the two-dimensional porous medium equation (2D PME). The numerical results obtained based on the tested examples 1, 2 and 3 show that the application of the Newton-SOR iterative method, which uses the optimal relaxation parameter, able to reduce the number of iterations and computer time for solving the 2D PME(see in Tables 1, 2, 3 and 4). Besides that, the accuracy of the numerical solutions by the Newton-SOR iterative method is better than the Newton-GS iterative method. It can be seen in Tables 1, 2 and 3 that the maximum absolute errors produced by the Newton-SOR iterative method is smaller than by the Newton-GS iterative method. This work will be extended to study the uses of two different relaxation parameters or the Modified SOR [12]. 


\section{ACKNOWLEDGEMENTS}

The authors would like to acknowledge the financial support received in the form of fundamental research grant scheme (GUG0022-SG-M-1/2016) from University Malaysia Sabah, Malaysia.

\section{REFERENCES}

[1] PolyaninA D, ZhurovA I,Vyaz'minA V. Exact solutions of nonlinear heat and mass transfer equations. Theoretical Foundations of Chemical Engineerin, 2000, 34(5):403-415

[2] Polyanin A. D.,Zaitsev V. F.Handbook of nonlinear partial differential equations.Florida: CRC Press, 2004

[3] BertelliF, FariaJ D, GoulartP R, BritoC, CheungN, Garcia A. Numerical and experimental modeling of two-dimensional unsteady heat transfer during inward solidification of square billets. Applied Thermal Engineering, 2016, 96:454-462

[4] BoranaR, Pradhan V, Mehta M. Numerical solution of instability phenomenon arising in double phase flow through inclined homogeneous porous media. Perspectives in Science, 2016, 8:225-227

[5] PamukS, Pamuk N. Solution of two-dimensional heat and mass transfer equation with power-law temperature-dependent thermal conductivity.TWMS Journal of Applied and Engineering Mathematics, 2014, 4(2):199-208

[6] ChewJ V L, Sulaiman J. Implicit solution of 1D nonlinear porous medium equation using the four-point Newton-EGMSOR iterative method.Journal of Applied Mathematics and Computational Mechanics,2016, 15(2):11-21

[7] ChewJ V L,Sulaiman J. Half-sweep Newton-Gauss-Seidel for implicit finite difference solution of 1D nonlinear porous medium equations.Global Journal of Pure and Applied Mathematics, 2016, 12(3):2745-2752

[8] Ortega J M,Rheinboldt W C. Monotone iterations for nonlinear equations with application to Gauss-Seidel methods. SIAM Journal on Numerical Analysis, 1967, 4(2):171-190

[9] Moré JJ. Global convergence of Newton-Gauss-Seidel methods. SIAM Journal on Numerical Analysis, 1971, 8(2):325-336

[10] YoungD M. Iterative methods for solving partial difference equations of elliptic 
type.Transactions of the American Mathematical Society, 1954, 76(1):92-111

[11] SommeijerB P, van der HouwenP J. Software with low storage requirements for two-dimensional nonlinear, parabolic differential equations.ACM Transactions on Mathematical Software, 1984, 10(4):378-396

[12] Kincaid D R, Young D M. The modified successive overrelaxation method with fixed parameters. Mathematics of Computation, 1972, 26(119):705-717

\section{How to cite this article:}

Chew J. V. L, Sulaiman J. Newton-SOR iterative method for solving the two-dimensional Porous Medium Equation. J. Fundam. Appl. Sci., 2017, 9(6S), 384-394. 